\title{
Ginzburg-Landau equation for steps on creep curve
}

\author{
Mulugeta Bekele ${ }^{1, *}$ and G. Ananthakrishna ${ }^{2}$ \\ ${ }^{1}$ Department of Physics, ${ }^{2}$ Materials Research Centre \\ Indian Institute of Science, Bangalore 560 012, India
}

\begin{abstract}
We consider a model proposed earlier by us for describing a form of plastic instability found in creep experiments. The model consists of three types of dislocations and some transformations between them. The model is known to reproduce a number of experimentally observed features. The mechanism for the phenomenon has been shown to be Hopf bifurcation with respect to physically relevant drive parameters. Here, we present a mathematical analysis of adiabatically eliminating the fast mode and obtaining a Ginzburg-Landau equation for the slow modes associated with the steps on creep curve. The transition to the instability region is found to be one of subcritical bifurcation over most of the interval of one of the parameters while supercritical bifurcation is found in a narrow mid-range of the parameter. This result is consistent with experiments. The dependence of the amplitude and the period of strain jumps on stress and temperature derived from the Ginzburg-Landau equation are also consistent with experiments. On the basis of detailed numerical solution via power series expansion, we show that high order nonlinearities control a large portion of the subcritical domain.
\end{abstract}

\footnotetext{
*On leave from Department of Physics, Addis Ababa University, Addis Ababa, Ethiopia
} 


\section{Introduction}

Instabilities in plastic flow has been an object of attention for a long time in metallurgical literature. Conceptually simplest form of this [Lubahn \& Felgar, 1961; Hall, 1970] manifests when the material is subjected to a creep test wherein a force is applied and the response in the form of elongation of the material is measured, which under normal conditions, is a smooth strain-time curve. However, under certain metallurgical conditions, one sees steps on creep curve suggesting a form of instability [Lubahn \& Felgar, 1961; Hall, 1970; Da Silveira \& Monteiro, 1979]. A better known form of the instability having the same physical origin, but conceptually more difficult, appears when the material is deformed under tensile deformation [Bodner \& Rosen, 1967; Brindley \& Worthington, 1970; Penning, 1972]. Here, the material is subjected to a predetermined response namely a constant rate of deformation, and the force or the stress developed in the sample is sought to be measured. Even in this case, one finds a smooth stress-strain curve under normal conditions. However, when the system is in the regime of instability, for some values of material parameters the stress-strain curve exhibits multiple load drops. Each of the load drops is related to the formation and propagation of dislocation bands [Chihab et al, 1987]. It is in the latter type of testing where plastic instability manifests much more easily than in the former. The phenomenon is referred to as the Portevin-Le Chatelier (PLC) effect or the jerky flow and is seen in several metals such as commercial aluminium, brass, alloys of aluminium and magnesium [Brindley \& Worthington, 1970]. It is observed only in a window of strain rates and temperature. In contrast, the phenomenon of steps on creep curve, which is the subject of the present discussion, is seen in few instances [Da Silveira \& Monteiro, 1979; Zagarukuyko et al, 1977; Stejskalova et al, 1981 ]. The reason attributed to this is that it is difficult to obtain proper control on metallurgical parameters wherein this form of instability can be observed. There is one more form of instability which manifests under constant stress test where similar strain jumps are seen as a function of time. The origin of instability in all these three modes of testing is known to be the same.( For a current status of both experiments and theory see Kubin et al, 1993). Eventhough steps on creep curve are seen in a limited number of experiments, it is often straight forward to translate many of the experimental results of constant strain rate test into that of creep test. It is generally agreed that the microscopic origin of the instabilities arises due to the interaction of dislocations with mobile point defects and is referred to as dynamic strain ageing. This leads to negative strain rate characteristic. The basic idea was formulated by Cottrell [1953] few decades ago.

The early phenomenological models are all static since they do not deal with time development. In contrast, methodology of dynamical systems addresses precisely this aspect. Until about a decade and a half ago, there were no models which looked at the problem from the point of view of bifurcation theory. An attempt to understand the problem in the above perspective was first made by our group several years ago [Ananthakrishna \& Sahoo, 1981b; Ananthakrishna \& Valsakumar, 1982, 1983; Valsakumar \& Ananthakrishna, 1983]. In a series of papers starting from an extended Fokker-Planck equation for the distribution function of the velocity of dislocation segments, and then splitting this into a mobile and an immobile component, we arrived at a model which consisted of three types of dislocations and some transformations between them [Ananthakrishna \& Sahoo, 1981a, 1981b; Sahoo \& Ananthakrishna, 1982; Valsakumar \& Ananthakrishna, 1983]. The basic idea could be

summarized by stating that the phenomena is due to a Hopf bifurcation resulting from nonlinear interactions between three different types of dislocations, suggesting a new mathematical mechanism for the instability. Eventhough the spatial inhomogeneous structure 
was ignored and only the temporal oscillatory state was sought to be described, the model and its extensions to the case of constant strain rate test, proved to be very successful in that it could explain most of the experimentally observed features (for critical reviews see Kubin \& Lepinoux, 1988 and Kubin et al, 1993). For instance, the existence of negative strain rate sensitivity in a regime of strain rates, comes out naturally as a consequence of the Hopf bifurcation [Ananthakrishna \& Valsakumar, 1982]. It must be emphasized, that this feature has been measured in most experiments on the PLC effect, and is assumed in all other theoretical models [ Cottrell, 1953; Bodner \& Rosen, 1967; Penning, 1972; Kubin and Estrin 1990; see also Kubin \& Martin, 1988; Martin \& Kubin, 1992; Kubin et al 1993]. Other experimentally observed features such as the existence of bounds on strain rate for the PLC effect to occur, the existence of critical strain and its dependence on applied strain rate, the dependence of the amplitude on the strain etc., also follow. One other important prediction which is direct consequence of the dynamical basis of the model is the existence of chaotic stress drops in a window of strain rates [Ananthakrishna \& Valsakumar, 1983; Ananthakrishna \& John, 1990]. Recently this prediction has been verified which in turn implies that only a few degrees of freedom are required for a dynamical description of the phenomenon. This offers justification for the use of only a few degrees of freedom for the description of the temporal aspect, eventhough the system is spatially extended. [Ananthakrishna et al., 1995; Ananthakrishna \& Noronha, 1995; Quaouire \& Fressengeas, 1995; Venkadesan et al 1995; Noronha et al 1996 \& 1997]. (Note that a spatially extended system implies infinite degrees of freedom.) Description of the phenomenon which includes the initiation and propagation of the bands during the PLC effect has also been recently attempted [Ananthakrishna, 1993].

Since the introduction of bifurcation theory into this field several years ago by our group, there has been a resurgence of interest in plastic instabilities in the light of introduction of new methodology borrowed from the theory of dynamical systems. This has further helped to obtain new insights hitherto not possible [Kubin \& Martin, 1988; Estrin \& Kubin, 1989; Kubin \& Estrin, 1990; see also Kubin \& Martin, 1988; Martin \& Kubin, 1992; Kubin, 1993; Ananthakrishna et al., 1995a \&b and references therein]. One of the aims of such theories is to be able to relate the microscopic dislocation mechanisms to the measurable macroscopic quantities. However, in the process, we feel that finer aspects of dynamical systems have been glossed over in this field. For instance, one often finds that casual remarks are made about fast and slow modes without actually going through the procedure of demonstrating the existence of such modes and eliminating the fast modes in favour of the slow ones [Aifantis, 1988; Hahner \& Kubin, 1992; Hahner, 1993]. In addition, under the adiabatic elimination, the slow modes are complicated functions of the original modes. Yet, hand waiving arguments have been used in building models which we believe are technically suspect.

The purpose of this article is at least three fold. First, we wish to illustrate the power and utility of dynamical methods to the study of dynamical aspects of the model. We demonstrate the technical aspects of adiabatic elimination (largely addressed to metallurgists working in the area of plastic instabilities) and then derive the equation for the slow modes having the form of time-dependent Ginzburg-Landau (TDGL) equation. Second, we wish to compare the results of the dependence of the amplitude and the period of the strain jumps on stress and temperature with experiments on creep curve as also with the results translated from the constant strain rate case to the creep case. This will allow us to relate the theoretically introduced parameters to the macroscopically measured quantities. Third, we wish to compare these results and the detailed numerical results via power series expansion with the approximate solutions for the limit cycles obtained earlier [Ananthakrishna \& Sahoo, 1981b; Valsakumar \& Ananthakrishna, 1983]. 
The principal results of this work are as follows. We show that the derived quintic TDGL equation for the slow modes is valid in the mid-range of the instability domain. Within this range, there is a narrow sub-range of the parameter where the system exhibits a supercritical bifurcation, beyond which subcritical bifurcation is exhibited. The results of this calculations on the amplitude and period of the strain jumps are compared with experiments. In addition, analytical work based on the TDGL equation and a detailed comparative numerical study via the power series solution shows that the model exhibits unusual properties in a certain domain of the parameter. The study further shows that very high order nonlinearities actually govern the nature of the subcritical bifurcation beyond the domain handled by the quintic TDGL equation.

The plan of the paper is as follows. In what follows (section 2) we present a brief summary of the model. In section 3, the technique of extracting the TDGL equation using reductive perturbative approach is applied to the model. In section 4, approximate limit cycle solution obtained through the TDGL equation is compared with experiments and with the numerical solution of the model as well as earlier studies on the model. Section 5 contains summary and discussion.

\section{A Model for Steps on Creep Curve}

We start with a brief summary of the model. The basic idea of the model is that instabilities in plastic flow are a consequence of nonlinear interactions between different types of dislocation populations. Spatial dependence is ignored and only temporal aspects are described, the idea being that the few degrees of freedom used for describing the phenomenon (dislocation populations) correspond to the collective modes of dislocations in the spatially extended system. As mentioned in the introduction, this finds support from the recent experimental verification of chaos in the PLC effect. The details of the model can be found in the original references [Ananthakrishna \& Sahoo, 1981b; Valsakumar \& Ananthakrishna, 1983]. The model consists of mobile dislocations $m$ and immobile dislocations $i m$ and another type which mimics the Cottrell's type $i$, which are dislocations with clouds of solute atoms. Let the corresponding densities be $N_{m}, N_{i m}$ and $N_{i}$, respectively. The basic dislocation mechanisms included are the following: (a) production of dislocations by cross glide $\left(m \stackrel{\theta V_{m}}{\rightarrow} m+m\right.$ , $\theta$ is the cross glide coefficient, $V_{m}$ is the velocity of mobile dislocations; $V_{m}=V_{0}\left(\sigma_{a} / \sigma_{0}\right)^{m}$ [Alexander, 1986] where $\sigma_{a}$ is the applied stress), (b) immobilization of two mobile dislocations $(m+m \stackrel{\beta}{\rightarrow} i m+i m, \beta$ is the rate at which two mobile dislocations get immobilized) and annihilation of two mobile dislocations $\left(m+m \stackrel{\beta^{\prime}}{\rightarrow} 0\right)$, and annihilation of a mobile with an immobile dislocation $(m+i m \stackrel{\bar{\beta}}{\rightarrow} 0)$, (c) once a mobile dislocation is immobilized, it can be reactivated by athermal or thermal means $(i \stackrel{\gamma}{\rightarrow} m)$, (d) lastly, a mobile dislocation can acquire a cloud of solute atoms and move with them. We consider such dislocations as distinct from both the mobile as well as the immobile and denote them $i$. This process is represented by $m \stackrel{\alpha_{m}}{\rightarrow} i, \alpha_{m}$ being a function of the concentration of solute atoms. However, as the solute atoms gather progressively around dislocations, these dislocations eventually will be immobilized $\left(i \stackrel{\alpha_{i}}{\rightarrow} i m\right)$. ( Note that in principle, $\beta, \beta^{\prime}$ and $\bar{\beta}$ are different.) These mechanisms lead to the rate equations for the densities of dislocations:

$$
\begin{aligned}
\dot{N}_{m} & =\theta V_{m} N_{m}-\left(\beta+\beta^{\prime}\right) N_{m}^{2}-\bar{\beta} N_{m} N_{i m}+\gamma N_{i m}-\alpha_{m} N_{m} \\
\dot{N}_{i m} & =\beta N_{m}^{2}-\bar{\beta} N_{i m} N_{m}-\gamma N_{i m}+\alpha_{i} N_{i}, \\
\dot{N}_{i} & =\alpha_{m} N_{m}-\alpha_{i} N_{i} .
\end{aligned}
$$


(For the sake of simplicity, we take $\beta^{\prime}=0$ and $\bar{\beta}=\beta$.) One can give a more transparent interpretation for Eq. (3) which represents whole process of acquiring a cloud of solute atoms by a mobile dislocation and eventual immobilization. This can be seen by defining $N_{i}=\int_{-\infty}^{t} K\left(t-t^{\prime}\right) N_{m}\left(t^{\prime}\right) d t^{\prime}$, where the kernel $K(t)$ which represents the fact that solute atoms are arriving at a certain rate has been chosen to be $\alpha_{m} e^{-\alpha_{i} t}$. It can be easily checked that Eq. (3) is the differential form of the definition of $N_{i}$. One last remark about some of the terms appearing in these equations. The first two terms plus the last term in Eq.(1), and equivalent terms in Eqs.(2), were derived starting from a velocity distribution function of the dislocation segments [ Ananthakrishna \& Sahoo 1981a; Sahoo \& Ananthakrishna, 1982]. Thus, this model is an improved model to explain the instability in plastic flow. Equations (1-3) can be cast into a dimensionless form by using scaled variables:

$$
x=N_{m}\left(\frac{\beta}{\gamma}\right), y=N_{i m}\left(\frac{\beta}{\theta V_{m}}\right), z=N_{i}\left(\frac{\beta \alpha_{i}}{\gamma \alpha_{m}}\right), \tau=\theta V_{m} t
$$

to get

$$
\begin{aligned}
\dot{x} & =(1-a) x-b_{0} x^{2}-x y+y, \\
\dot{y} & =b_{0}\left(b_{0} x^{2}-x y-y+a z\right), \\
\dot{z} & =c(x-z),
\end{aligned}
$$

where $a=\frac{\alpha_{m}}{\theta V_{m}}, b_{0}=\frac{\gamma}{\theta V_{m}}$, and $c=\frac{\alpha_{i}}{\theta V_{m}}$. The dot represents differentiation with respect to $\tau$. Equations (5-7) are coupled set of nonlinear equations which support limit cycle solutions for a range of parameters $a, b_{0}$ and $c$, that are physically relevant. $a$ refers to the concentration of the solute atoms, $b_{0}$ refers to the reactivation of immobile dislocations and $c$ to the time scales over which the slowing down occurs. We have demonstrated the existence of limit cycle solutions and also obtained approximate closed form solutions for the limit cycles [Ananthakrishna \& Sahoo, 1981b; Valsakumar \& Ananthakrishna, 1983]. In addition, the model has been studied numerically. Using the Orowan equation which relates the rate of change of $\operatorname{strain}(\dot{S})$ to mobile dislocation density and the mean velocity: $\dot{S}=b N_{m} V_{m}$, with $b$ as the Burger's vector, steps on the creep curve follow automatically since the densities of dislocations are oscillatory. Several experimental results are reproduced [Ananthakrishna \& Sahoo, 1981b; Valsakumar \& Ananthakrishna, 1983].

\section{Reductive Perturbative Approach}

For the sake of completeness, we outline the reductive perturbative approach. Near the point of Hopf bifurcation of the system (Eqs.5-7), corresponding to a critical value of the drive parameter, a pair of complex conjugate eigenvalues and another real negative eigenvalue exist for the linearized system of equations around the steady state. As we approach the critical value from the stable side, the real parts of the pair of complex conjugate eigenvalues approach zero and hence the corresponding eigenvectors get slower and slower. In contrast, the effect of the change in the drive parameter on the real negative eigenvalue is negligible. Thus, the eigenvector corresponding to the real negative eigenvalue is the fast mode. For this reason, the slow modes are the ones which determine the formation of new states of order. The reductive perturbative method is a method where the asymptotic equation is extracted in a systematic way [Taniuti \& Wei, 1968; Newell \& Whitehead, 1969; Kuramoto \& Tsuzuki, 1974; Mashiyama et al., 1975; Richter et al., 1981]. The method involves in first finding 
the eigenvectors corresponding to the slow modes and looking for a solution in the subspace spanned by these vectors. The effect of the nonlinearity is handled progressively as a perturbation of the linear solution in power series of the deviation from the critical value of the drive parameter. It may be worth emphasizing that this method is essentially the same as reduction to center manifold. Indeed, the equivalence of the center manifold theory [Carr, 1981; Guckenheimer \& Holmes, 1983; Troger \& Steindl, 1991] with the reductive perturbation has been established [Chen et al, 1996]. Other techniques of extracting amplitude equations have been devised whose end results are basically the same. For instance, perturbative renormalization group method [Goldenfeld et al 1989; Chen et al, 1996] and its recent extension on the basis of envelope theory [Kunihiro, 1995 \& 1996] has also been developed as a tool for global asymptotic analysis which can be used to extract the amplitude equations.

In the present model, there is one simplification arising from the fact that $x$ can be identified as the fast (stable) mode, and hence this will be eliminated adiabatically. Then we derive the equation for the slow dynamics through a power series expansion. Although this adiabatic elimination is approximate, the result is found to be in a reasonably good agreement with the accurate method (which will be reported elsewhere) except for finer details.

Consider the Eqs.(5-7). It can be shown that there is a domain of instability for the parameters $a, b_{0}$ and $c$. The range of values of $b_{0}$ is $\sim 0-10^{-2}$, that of $c$ is $\sim 0-10^{-1}$ while $a \sim 1$. Thus, $b_{0}$ and $c \ll 1$. Thus, there are three time scales that are in principle different from each other. Physically, they correspond to the mobile (fastest $a$ ), the Cottrell type $(c)$ and the immobile (slowest $b_{0}$ ). Under these conditions we can eliminate one full equation adiabatically. This aspect becomes transparent if the above equations are written in terms of variables which are deviations from the steady state. There is only one steady state defined by:

$$
x_{a}=z_{a}=\frac{1-2 a+\left[(1-2 a)^{2}+8 b_{0}\right]^{1 / 2}}{4 b_{0}}, \text { and, } y_{a}=1 / 2 .
$$

Defining new variables which are deviations from the steady state

$$
X=x-x_{a}, Y=y-y_{a}, \text { and } Z=z-z_{a},
$$

Eqns. (5-7) take the form

$$
\begin{aligned}
\dot{X} & =-\left(\alpha X+\chi Y+b_{0} X^{2}+X Y\right), \\
\dot{Y} & =-b_{0}\left(\Gamma X+\delta Y-a Z-b_{0} X^{2}+X Y\right), \\
\dot{Z} & =c(X-Z) .
\end{aligned}
$$

where

$$
\begin{array}{r}
\alpha=a+2 b_{0} x_{a}+y_{a}-1, \chi=x_{a}-1, \\
\Gamma=y_{a}-2 b_{0} x_{a}, \delta=x_{a}+1 .
\end{array}
$$

Now, we can rescale the time-like variable by $\tau^{\prime}=b_{0} \tau$ and get

$$
\begin{aligned}
b_{0} \frac{d X}{d \tau^{\prime}} & =-\left(\alpha X+\chi Y+b_{0} X^{2}+X Y\right), \\
\frac{d Y}{d \tau^{\prime}} & =-\left(\Gamma X+\delta Y-a Z-b_{0} X^{2}+X Y\right), \\
\frac{d Z}{d \tau^{\prime}} & =\frac{c}{b_{0}}(X-Z) .
\end{aligned}
$$


It is clear that in the limit of small $b_{0},|X| \rightarrow \infty$, unless the left hand side vanishes identically. Thus, we can eliminate $X$ in favour of the other two variables and obtain:

$$
\begin{aligned}
\frac{d Y}{d \tau} & =-b_{0}\left[a X+2\left(x_{a}+X\right) Y-a Z\right], \\
\text { and } \frac{d Z}{d \tau} & =c[X-Z],
\end{aligned}
$$

where

$$
X=\frac{1}{2 b_{0}}\left(-(\alpha+Y)+\left[Y^{2}+2\left(\alpha-2 b_{0} \chi\right) Y+\alpha^{2}\right]^{\frac{1}{2}}\right) .
$$

(The other root for $X$ is unphysical since it corresponds to negative dislocation density.) It must be emphasized that this adiabatic elimination becomes more exact as the value of the parameter $b_{0}$ gets smaller. Equations (17-18) will be solved reductive perturbatively. Writing these equations as a matrix equation where the nonlinear part appears separately from the linear part, we get

$$
\frac{d \vec{R}}{d \tau}=\mathbf{L} \vec{R}+\vec{N}
$$

where

$$
\begin{gathered}
\vec{R}=\left(\begin{array}{c}
Y \\
Z
\end{array}\right), \\
\mathbf{L}=\left(\begin{array}{cc}
c_{0} & a b_{0} \\
-\frac{c \chi}{\alpha} & -c
\end{array}\right),
\end{gathered}
$$

with $c_{0}=b_{0}\left(\frac{a \chi}{\alpha}-2 x_{a}\right)$ and the nonlinear part, $\vec{N}$, is given by

$$
\vec{N}=\left(\begin{array}{c}
-a b_{0}\left(X+\frac{\chi}{\alpha} Y\right)-2 b_{0} X Y \\
c\left(X+\frac{\chi}{\alpha} Y\right)
\end{array}\right)=\left(\begin{array}{c}
\sum_{n=2}^{\infty} \xi_{n} Y^{n} \\
\sum_{n=2}^{\infty} \zeta_{n} Y^{n}
\end{array}\right)
$$

The coefficients $\xi_{n}$ and $\zeta_{n}$ appearing in the last expression for $\vec{N}$ are functions of $a, b_{0}$ and $c$ whose expressions for the required first few are given in Eqs.(A8-A15) of Appendix A.

Consider stability of the fixed point as a function of the parameter $c$. The eigenvalues, $\lambda_{ \pm}$, of the matrix $\mathbf{L}$ are

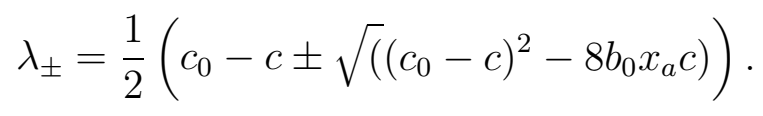

The fixed point becomes unstable when $c$ is less than $c_{0}$ ( $c$ is non-negative) and the discriminant of Eq.(24) is negative giving a pair of complex conjugate eigenvalues. This holds when the inequality $(3 a-1)\left(1-2 a^{2}\right) \geq 2 b_{0}(2+a)^{2}$ is satisfied. The instability region in the $\frac{1}{c}$ versus $a$ plane for a fixed value of $b_{0}\left(=10^{-4}\right)$ is shown in Fig. 1. It extends approximately between $a=\frac{1}{3}$ and $a=\frac{1}{\sqrt{2}}$.

To get approximate analytical solution of Eq.(20), we follow the reductive perturbative approach similar to that used by Mashiyama et al. [1975] and Richter et al. [1981]. We choose $c=c_{0}(1-\epsilon)$ with $0<\epsilon \ll 1$ and write the matrix $\mathbf{L}$ as a sum of two matrices, $\mathbf{L}=\mathbf{L}_{0}+\epsilon \mathbf{L}_{1}$, where $\mathbf{L}_{0}$ is the matrix $\mathbf{L}$ evaluated for $c=c_{0}$ and

$$
\mathbf{L}_{1}=\left(\begin{array}{cc}
0 & 0 \\
\frac{c_{0} \chi}{\alpha} & c_{0}
\end{array}\right)
$$


The eigenvalues of $\mathbf{L}_{0}$ are $\lambda_{0 \pm}= \pm \imath \omega$, where $\left.\omega=\sqrt{(} 2 b_{0} c_{0} x_{a}\right)$. Considering that the solution for $\vec{R}$ grows continuously out of the critical eigenmodes, we can express it as

$$
\vec{R}(\tau)=\Psi(\tau) e^{\imath \omega \tau} \overrightarrow{r_{+}}+\Psi^{*}(\tau) e^{-\imath \omega \tau} \overrightarrow{r_{-}}
$$

where $\Psi$ and $\Psi^{*}$ are time-dependent complex amplitudes while $\vec{r}_{ \pm}$are right eigenvectors defined by $\mathbf{L}_{0} \vec{r}_{ \pm}= \pm \imath \omega \vec{r}_{ \pm}$. Similarly, we introduce left eigenvectors, $\vec{s}_{ \pm}^{T}$ defined by $\vec{s}_{ \pm}^{T} \mathbf{L}_{0}=$ $\pm \imath \omega \vec{s}_{ \pm}^{T}$. (Note that $\vec{s}_{+}^{T} \vec{r}_{-}=\vec{s}_{-}^{T} \vec{r}_{+}=0$. T refers to transpose operation of the matrix concerned). Substituting this expression for $\vec{R}$ in Eq.(20) and multiplying it by $\vec{s}_{+}^{T}$ from the left-hand side gives the equation governing $\Psi$ :

$$
e^{i \omega \tau} \frac{d \Psi}{d \tau}=\epsilon \mu \Psi e^{i \omega \tau}-\epsilon \mu^{*} \Psi^{*} e^{-i \omega \tau}+\sum_{n=2}^{\infty} P_{n}\left(\psi e^{i \omega \tau}+\text { c.c. }\right)^{n} .
$$

$\mu=\mu_{1}+\imath \mu_{2}$ and $P_{n}$ are complex coefficients which are functions of $a, b_{0}$ and $c$. Their expressions are, respectively, given in Eqs. (A1) and (A4) in Appendix A. We express $\Psi$ in power series of $\epsilon^{\frac{1}{2}}$ :

$$
\Psi=\epsilon^{\frac{1}{2}} \psi_{1}+\epsilon \psi_{2}+\ldots
$$

and introduce slowly varying multiple time scales $\tau_{1}(=\epsilon \tau), \tau_{2}\left(=\epsilon^{2} \tau\right), \ldots$ replacing $\frac{d}{d \tau}$ by $\frac{\partial}{\partial \tau}+\epsilon \frac{\partial}{\partial \tau_{1}}+\epsilon^{2} \frac{\partial}{\partial \tau_{2}}+\ldots$. With this substitution in Eq.(27), it can be solved successively by equating terms of equal power in $\epsilon$ on both sides of the equation. At first, $\mathcal{O}\left(\epsilon^{\frac{1}{2}}\right)$ terms give rise to the equation

$$
\frac{\partial \psi_{1}}{\partial \tau}=0
$$

which means that, on the time scale of $\tau, \psi_{1}$ is constant. The next higher order, $\mathcal{O}(\epsilon)$, terms give the equation

$$
\frac{\partial \psi_{2}}{\partial \tau}=P_{2}\left(\psi_{1} e^{i \omega \tau}+\text { c.c. }\right)^{2} e^{-i \omega \tau}
$$

which can be integrated to get $\psi_{2}$. $\mathcal{O}\left(\epsilon^{\frac{3}{2}}\right)$ terms give the equation

$\frac{\partial \psi_{3}}{\partial \tau}+\frac{\partial \psi_{1}}{\partial \tau_{1}}=\mu \psi_{1}-\mu^{*} \psi_{1}^{*} e^{-2 i \omega \tau}+\left[2 P_{2}\left[\left(\psi_{1} \psi_{2} e^{2 i \omega \tau}+\psi_{1} \psi_{2}^{*}\right)+\right.\right.$ c.c. $\left.]+P_{3}\left(\psi_{1} e^{i \omega \tau}+\text { c.c. }\right)^{3}\right] e^{-i \omega \tau}$,

from which we extract the slow dynamics:

$$
\frac{\partial \psi_{1}}{\partial \tau_{1}}+\mu \psi_{1}+\eta\left|\psi_{1}\right|^{2} \psi_{1}
$$

Since to $\mathcal{O}\left(\epsilon^{\frac{1}{2}}\right) \Psi=\epsilon^{\frac{1}{2}} \psi_{1}$, Eq. (32) gives the dynamics for the complex amplitude $\Psi$ :

$$
\frac{d \Psi}{d \tau}=\epsilon \mu \Psi+\eta|\Psi|^{2} \Psi
$$

The expression for the complex coefficient $\eta=\eta_{1}+\imath \eta_{2}$ is given in Eq. (A2) in Appendix A. Equation (33) is a cubic TDGL equation where $\Psi$ is the complex amplitude for the slow mode which is also referred to as complex order parameter (of the new state of temporal order). Its steady state solution gives the amplitude squared as

$$
|\Psi|^{2}=-\epsilon \frac{\mu_{1}}{\eta_{1}}
$$


and the associated frequency, $\Omega$, (with $\Psi=|\Psi| e^{\imath \Omega \tau}$ ) as

$$
\Omega=\epsilon\left(\mu_{2}-\frac{\eta_{2}}{\eta_{1}} \mu_{1}\right) .
$$

Note that $\Psi$ describes the limit cycle oscillation. This solution exists provided $\eta_{1}$ is negative since $\mu_{1}$ is positive (see expression of $\mu$ in Eq.(A1) in Appendix A). $\eta_{1}$ is found to be negative in a narrow region $(0.4713 \leq a \leq 0.5120)$ as can be seen in Fig. 2. In this case, since the amplitude of the slow modes grows continuously in proportion to $\epsilon^{\frac{1}{2}}$ (see Eq. (34)) the transition is supercritical bifurcation (continuous or 'second order') For other values of $a$ (within the instability range), $\eta_{1}$ is positive implying that the transition is subcritical bifurcation (discontinuous or 'first order'). One has to then go to quintic or even higher terms in the TDGL equation for obtaining an expression for the slow modes (order parameter). We have carried out the reductive perturbative method further to derive the quintic TDGL equation:

$$
\frac{d}{d \tau} \Psi=\epsilon \mu \Psi+\eta|\Psi|^{2} \Psi+\nu|\Psi|^{4} \Psi
$$

The steady state solution of this equation gives the amplitude squared as

$$
|\Psi|^{2}=\frac{1}{2}\left(-\frac{\eta_{1}}{\nu_{1}}+\sqrt{\left(\frac{\eta_{1}}{\nu_{1}}\right)^{2}-4 \epsilon \frac{\mu_{1}}{\nu_{1}}}\right),
$$

and the frequency as

$$
\Omega=\left(\eta_{2}-\eta_{1} \frac{\nu_{2}}{\nu_{1}}\right)|\Psi|^{2}+\epsilon\left(\mu_{2}-\mu_{1} \frac{\nu_{2}}{\nu_{1}}\right)
$$

We found $\nu_{1}$ to be negative in the range $0.4538 \leq a \leq 0.5097$ (see Fig. 2). This means a narrow region: $0.4538 \leq a \leq 0.4713$ within the subcritical bifurcation exists over which the quintic TDGL equation has a solution (see Fig. 2).

\section{Comparison with Experiments and Numerical Solu- tions}

\subsection{Comparison with Experiments}

To start with, consider the supercritical regime where expressions for the amplitude and period of the limit cycle are simple. Using the steady state solution of the cubic TDGL equation, Eqs.(34) \& (35), the dependence of $|\Psi|^{2}$ and period, $P \sim \frac{1}{(\omega+\Omega)}$, of the limit cycle on $a$ are, to the leading order, found to be $a^{-2}$ and (const. $\left.+a^{2}\right)$, respectively. The parameter $a(=$ $\left.\alpha_{m} / \theta V_{m}\right)$ is a function of the applied stress $\left(\sigma_{a}\right)$ and temperature $(\mathrm{T})$. As remarked earlier, $\alpha_{m}$ is proportional to the concentration of the solute atoms, and therefore $\alpha_{m} \sim \exp (-E / k T)$. Using the standard expression [ Alexander 1986] $V_{m}\left(\sigma_{a}, T\right)=V_{0}\left(\sigma_{a} / \sigma_{0}\right)^{m} \exp \left(-E_{m} / k T\right)$ (with $m>1$ ), we get

$$
\frac{1}{a} \sim\left(\frac{\sigma_{a}}{\sigma_{0}}\right)^{m} e^{\frac{E-E_{m}}{k T}}
$$

From this we see that the amplitude of the limit cycle has a strong increasing dependence on stress and an increasing dependence on temperature if we assume that $E>E_{m}$. In contrast, the period of the limit cycle has a weak decreasing dependence on stress (as the leading contribution is constant) and a decreasing dependence on temperature. Since stress and 
temperature are measurable quantities, our predictions can be compared with experimental results. The amplitude and period of the limit cycle are related, respectively, to the amount of strain jumps and the period of the jumps on the creep curve through the Orowan equation. There are very few experiments in this mode of testing as mentioned in the introduction. The only experiment where this dependence on stress and temperature has been measured is that by Zagoruyko et al [1977]. However, it is possible to translate the results from experiments in constant strain rate case to the creep case and compare them with the results of this calculation. According to Zagoruyko et al [1977], the amplitude of the strain jumps increases with stress while its period has a weak but decreasing dependence on stress. Experiments from constant strain rate case also exhibit the same trend when the results are translated in terms of constant stress experiments. It is well known that the amplitude of the stress drops decreases with applied strain rate. In fact, even the present model predicts this behaviour for the constant strain rate mode [Ananthakrishna \& Valsakumar 1982]. This implies that the dependence of the amplitude of strain jumps on stress should increase [ Kubin et al 1993]. [ This relation can be seen as follows. In constant strain rate case, the deformation rate is fixed and the stress developed in the sample is measured. When the contribution to the plastic strain rate increases due to increased dislocation motion (for whatever reasons), the stress has to fall in order to keep the applied strain rate constant. Thus, the relation between strain rate and stress is opposite.] Clearly, the general trend is consistent with the experimental results. Fig. 3 shows the actual dependence of the amplitude and the period on $a$ in the domain of supercritical bifurcation (not the leading one as given by the above expressions). Zagarukuyko etal [1977] also report that the amplitude of the strain jumps increases while its period decreases with temperature, which is consistent with our result ( provided $E>E_{m}$ ).

In the case of subcritical bifurcation, the dependence of the amplitude of the strain jumps and the period on $a$ have similar behaviour as in the case of the supercritical bifurcation. Eventhough, in this case, leading order dependence on $a$ is difficult to obtain we have their actual dependence plotted in Fig. 4. It may be noted that they have qualitatively the same behaviour as in Fig. 3 with the degree of dependence more pronounced in this case.

Experiments in constant strain rate case show that the stress drops are seen to arise both abruptly as well as continuously [Kubin et al, 1988]. Translating this result to the constant stress case, it implies that the strain jumps can arise both abruptly and continuously. This feature again follows from our calculations.

\subsection{Comparison with Numerical Solutions}

Having derived the TDGL equation, we would first like to compare its result with the numerical solutions obtained via Eqs. (17-18) and via Eq.(20). Secondly, due to the fact that the quintic TDGL equation (Eq. 36) is valid in a limited domain, one suspects that higher order nonlinearities are controlling the subcritical bifurcation, which is quite unusual. Therefore, we would like to investigate the numerical solution obtained by solving Eq.(20) keeping successive leading powers in $Y$. This analysis confirms the above suspicion that higher order nonlinearities are important in this model outside the domain of validity of the quintic TDGL equation.

Using the steady state solution of the quintic TDGL equation, Eq.(36), in Eq.(26), we get an approximate expression for the limit cycle which is usually called the secular motion. The equations governing the secular motion are given by Eqs. (B5) and (B6) (hereafter called secular equations) in Appendix B. This can be compared with the numerical solutions 
obtained using Eqs. (17-18) (hereafter called the reduced model) and with that obtained using Eq. (20) where the expansion in $Y$ of Eq. (23) is limited up to a certain power (hereafter called the $Y^{n}$-truncated model, n signifying the highest power of $Y$ after truncation). At the outset we state that the reduced model has bounded solution over the entire instability domain.

In the case of the numerical solutions obtained from Eq.(20), we have found that while truncating the $Y$-expansion in Eq. (23) at $Y^{3}$ is sufficient to give a bounded solution for the range of supercritical bifurcation $(0.4713<a<0.512)$, it fails beyond this. On the other hand, truncating the $Y$-expansion at $Y^{5}$ does extend the domain of bounded solution $(0.4538<a<0.512)$ which matches with the domain where the quintic TDGL equation works.

Figure 5 shows the plots of the solutions to the secular equations (Eqs. B5 and B6), the reduced model (Eqs. 17-18) and the $Y^{3}$-truncated model (Eq. 20) for $a=0.5$ and $\epsilon=10^{-4}$. All of them are numerically almost indistinguishable. We have verified that the solutions obtained by these three methods show that the amplitude of the limit cycle scales with $\epsilon^{\frac{1}{2}}$.

Figure 6 shows the solutions obtained by the three ways for $a=0.468$ and $\epsilon=10^{-4}$ where, this time, the truncation is at $Y^{5}$. The agreement between the three solutions is reasonably good. Part of the discrepancy between the secular motion and the two numerical solutions can be attributed to the range of values of the various terms ( $k$ 's, $\ell$ 's, $m$ 's and $n$ 's of Appendix C) contributing to $\eta$ and $\nu$. (They range from $10^{-16}$ to $10^{9}$ ). We have also verified that the amplitude of the limit cycle has a finite jump for this case.

For $a<0.4538$, where the quintic TDGL equation is inadequate, $Y^{5}$-truncated model is also inadequate. However, $Y^{7}$-truncated model has a bounded solution for the region $0.4385<a<0.5120$. In particular, Fig. 7 shows the plots of the solutions obtained by the $Y^{7}$-truncated model and that by the reduced model for $a=0.44$ and $\epsilon=10^{-4}$. This implies that seventh order TDGL equation need to be considered for this range of a. We have verified that $Y^{9}$ term extends the domain of bounded solutions upto $a=0.4246$. From this trend, it appears that very high powers in $Y$ need to be retained to cover the entire domain upto $a=0.333$.

The region $a>0.5120$ is even more interesting. While the quintic TDGL equation is inadequate to give a bounded solution, we found that retaining even upto the ninth power in $Y$ does not give a bounded solution for the entire range of values $0.512<a<0.707$ (even for those close to $a=0.512)$. This suggests that this region is one where the nonlinearity is strong. On the other hand, as pointed out earlier the reduced model has limit cycle solution in this range as well. In particular, Fig. 8 shows such a plot for $a=0.63$ and $\epsilon=10^{-4}$.

Thus, the results of the TDGL equation, the $Y^{n}$-truncated model and the reduced model are consistent with each other. Further, the numerical solutions via the power series in $Y$ throws light on the nature of nonlinearities governing the solution in different regions of instability.

\section{$5 \quad$ Summary and Discussion}

We have carried out the reductive perturbative approach to the problem of steps on creep curve and shown that the dynamics of the system is described by a TDGL equation for the amplitude of the slow modes (complex order parameter) $\Psi$ in the neighbourhood of the bifurcation point. Since the above derivation is valid only in the neighbourhood of the critical value, the expression for $\Psi$ is valid only for small $\epsilon$. This has been exemplified by the 
reasonable agreement between the secular motion and the numerical solution of the reduced model within the domain of the validity of the quintic TDGL equation.

We have shown that both subcritical as well as supercritical bifurcations are seen in the instability range of the parameter $a$. While the subcritical bifurcation ( abrupt or 'first order' transition) seen over most of the values of a is consistent with our earlier calculation [Ananthakrishna \& Sahoo, 1981b; Valsakumar \& Ananthakrishna, 1983], we see a supercritical bifurcation ( continuous or 'second order' transition) over a narrow mid-range of a. The existence of the supercritical bifurcation was missed by us earlier. The existence of both supercritical and subcritical bifurcations is consistent with experiments. In addition, the dependence of the amplitude and the periodicity of the strain jumps on stress and temperature are consistent with experiments. From this point of view, even though the derived TDGL equation is valid in a limited domain, the present calculation allows a direct mapping of these quantities. This calculation also helps us to demonstrate the complicated dependence of the slow modes on the original modes. This will serve as a warning to those using hand waiving arguments for declaring certain modes as fast modes and others as slow modes in modelling of such of these problems.

Looked at from the point of view of the properties of the model, there are some interesting features. For the region outside the validity of the quintic TDGL equation, on the basis of a careful numerical analysis, we found that high order nonlinearities govern the nature of the subcritical bifurcation. It must be emphasized that this feature is unusual and this insight would not have been possible but for the numerical solutions obtained by keeping successive higher powers in $Y$, which in itself is the basis for the derivation of the TDGL equation.

This unusual feature of the model interpreted in the language of phase transitions give better insight and could also find applicability. For conventional models, the 'free energy' is described by an expansion in power series of the order parameter. Even in the case of dynamic transition such as the present one, it is possible to associate a 'free energy' like function such that $\frac{\partial \Psi}{\partial \tau}=-\frac{\delta F}{\delta \Psi^{*}}$, where $F\left[\Psi, \Psi^{*}\right]$ is the 'free energy' like function. While the 'free energy' for 'second order' phase transitions is described by retaining up to fourth power in the order parameter, up to sixth power is conventionally required for 'first order' phase transitions (with the appropriate signs for the coefficients in the expansion). In the present case, however, we need to go to as high as twelfth power (or more) of the order parameter to cover the interval $0.3333<a<0.4246$ (or equivalently twelfth power or higher in $Y$ ). In this case, including successively higher powers in $Y$ increases the domain of description. On the other hand, in the interval of $0.512<a<0.707$, even retaining upto tenth power in $Y$ (which is the highest power we have checked) does not work. This must be contrasted with the existence of bounded solution for the reduced model over the entire interval. This feature is rather unusual and, to the best of our knowledge, we are not aware of any other model which exhibits this property.

\section{Acknowledgement}

One of us (M.B.) would like to thank the International Program in Physical Sciences, Uppsala University (Sweden) for offering a fellowship to study at Indian Institute of Science. Part of this work has been supported by IFCPAR Grant No. 1108-1. 


\section{References}

Aifantis, E. C. [1988] "On the problem of dislocation patterning and persistent slip bands" in Non Linear Phenomena in Materials Science I, eds. Kubin, L. P. \& Martin, G. (Trans Tech, Switzerland), pp. 397-406.

Alexander, H. [1986] "Dislocations in covalent crystals" in Dislocations in Solids ed. Nabarro, F. R. N. (North-Holland), 151.

Ananthakrishna, G. [1993] "Formation, propagation of bands and chaos in jerky flow", Scripta. Metall. 29, 1183-1188.

Ananthakrishna, G. \& John, T. M. [1990] "Instabilities and chaos in plastic flow", in Directions in Chaos Vol. 3, ed. Hao Bai-lin (World Scientific, Singapore), pp. 133148.

Ananthakrishna, G., Fressengeas C., Grosbras, M., Vergnol, J., Engelke, C., Plessing, J., Neuhaeuser, H., Bouchaud, E., Planes, J. \& Kubin, L. P. [1995a] "On the existence of chaos in jerky flow," Scripta Metall. 32, 1731-1737.

Ananthakrishna, G., Kubin, L. P. \& Martin, G. (eds.) [1995b] Solid State Phenomena Vol. $42 \& 43$ (Scitec, Switzerland).

Ananthakrishna, G. \& Noronha, S. J. [1995] "Chaos in jerky flow: Theory and experiment" in Solid State Phenomena Vol. 42\&43, eds. Ananthakrishna, G., Kubin, L. P. \& Martin, G. (Scitec, Switzerland), pp. 277-286.

Ananthakrishna G. \& Sahoo, D. [1981a] "A statistical dislocation dynamics with application to LiF", J. Phys. D 14), 699-713.

Ananthakrishna, G. \& Sahoo, D. [1981b] "A model based on nonlinear oscillations to explain jumps on creep curves", J. Phys. D 14, 2081-2090.

Ananthakrishna, G. \& Valsakumar, M. C. [1982] "Repeated yield drop phenomena: a temporal dissipative structure", J. Phys. D 15, L171-L175.

Ananthakrishna, G. \& Valsakumar, M. C. [1983] "Chaotic flow in a model for repeated yielding", Phys. Lett. A 95, 69-71.

Ananthakrishna, G., Fressengeas C., Grosbras, M., Vergnol, J., Engelke, C., Plessing, J., Neuhaeuser, H., Bouchaud, E., Planes, J. \& Kubin, L. P. [1995] "On the existence of chaos in jerky flow," Scripta Metall. 32, 1731-1737.

Bodner, S. R. \& Rosen, A. [1967] "Discontinuous yielding of commercially-pure aluminium", J. Mech. Phys. Solids 15, 63-77.

Brindley, B. J. \& Worthington, P. J. [1970] "Yield-point phenomena in substitutional alloys", Metall. Reviews 145, 101-114.

Carr, J. [1981] Applications of Center Manifold Theory (Springer-Verlag, Berlin).

Chen, L. Y., Goldenfeld, N. \& Oono, Y., [1996] "The Renormalization Group and Singular Perturbations: Multiple- Scales, Boundary Layers and Reductive Perturbation Theory", Phys. Rev. E 54, 376-394. 
Chihab, K., Estrin, Y., Kubin, L. P. \& Vergnol, J. [1987] "The kinetics of the Portevin-Le Chatelier effect in an Al-5 at\% Mg alloy", Scripta. Metall. 21, 203-208.

Cottrell, A. H. [1953] "A note on the Portevin-Le Chatelier effect", Phil. Mag. 44, 829-832.

Da Silveira, T. L. \& S. N. Monteiro, S. N. [1979] "Jumps in the creep curve of austentic stainless steels", Met. Trans. A 10, 1795-1796.

Estrin, Y. \& Kubin, L. P. [1989] "Collective dislocation behavior in dilute alloys and the Portevin-Le Chatelier effect", J. Mech. Behaviour of Materials 2, 255-292.

Goldenfeld, N., Martin, O. \& Oono, Y. [1989] "Intermediate Asymptotics and Renormalization Group Theory", J. Sci. Comp. 4, 355.

Guckenheimer, J. \& Holmes, P. [1983] Nonlinear Oscillations, Dynamical Systems, and Bifurcations of Vector Fields vol. 42 of Applied Math. Sciences (Springer-Verlag, Berlin), 123-145.

Hahner, P. \& Kubin, L. P. [1992] "Coherent Propagative Structures in Plastic Deformation: A Theory of Ludlers Bands in Polycrystals" in Solid State Phenomena Vol. 3\&4, eds. Kubin, L. P. \& Martin, G. (Trans Tech, Switzerland), pp. 385-402.

Hahner, P. [1993] "Modelling the spatiotemporal aspects of the Portevin-Le Chatelier effect", Mater. Sci. and Eng. 164, 23-34.

Hall, E. O. [1970] Yield Point Phenomena in Metals and Alloys (Macmillan, London).

Kubin, L. P., Chihab, K. \& Estrin, Y. [1988] "The rate dependence of the Portevin-Le Chatelier effect", Acta Metall. 36, 2707-2718.

Kubin, L. P. \& Estrin, Y. [1990] "Evolution of dislocation densities and the critical conditions for the Portevin-Le Chatelier effect", Acta Metall. 38, 697-708.

Kubin, L. P. \& Estrin, Y. (organizers) [1993] "Viewpoint Set No. 21: Propagative instabilities", Scripta Metall. 29, 1147-1188.

Kubin, L. P. \& Lepinoux, J. [1988] "The dynamic Organization of dislocation structures", Strength of Metals and Alloys (ICSMA 8), vol. 1, eds. Kettunen, P. O., Lepisto, T. K., \& Lehtonen, M. E., (Pergamon Press ,Oxford), pp. 35-59.

Kubin, L. P. \& Martin, G. (eds.) [1988] Solid State Phenomena Vol. 3\&4 (Trans Tech, Switzerland).

Kunihiro, T. [1995] "A Geometrical Formulation of the Renormalization Group Method for Global Analysis", Prog. Theor. Phys. 94 503-514.

Kunihiro, T. [1996] "The Renormalization Group Method Applied to Asymptotic Analysis of Vector Fields", in hep-th/9609045.

Kuramoto, Y. \& Tsuzuki, T. [1974] "Reductive perturbative approach to chemical instabilities", Prog. Theor. Phys. 52, 1399-1401.

Lubahn, J. D. \& Felgar, R. P. [1961] Plasticity and Creep of Metals (John Wiley, New York). 
Martin, G. \& Kubin, L. P. (eds.) [1992] Solid State Phenomena Vol.23\&24 (Trans Tech, Switzerland).

Mashiyama, H., Ito, A. \& Ohta, T. "Fluctuations and phase transitions far from equilibrium", Prog. Theor. Phys. 54, 1050-1066.

Newell, A. C. \& Whitehead, J. A. [1968] "Finite bandwidth, finite amplitude convection", J. Fluid Mech. 38, 279-303.

Noronha, S. J., Ananthakrishna, G., Quaouire, L. \& Fressengeas, C. [1997] "Chaos in Jerky Flow- Experimental verification of a theoretical prediction", Pramana, in press.

Noronha, S. J., Ananthakrishna, G., Quaouire, L., Fressengeas, C. \& Kubin, L. P. [1996] "Chaos in the Portevin-Le Chatelier Effect", submitted to Int. J. Bif. \& Chaos.

Penning, P. [1972] "Mathematics of the Portevin-Le Chatelier effect", Acta Metall. 20, 1169-1175.

Quaouire, L. \& Fressengeas, C. [1995] "Dynamical analysis of the Portevin-Le Chatelier effect" in Solid State Phenomena Vol. 3\&4 (Trans Tech, Switzerland), eds. Ananthakrishna, G., Kubin, L. P. \& Martin, G. (Scitec, Switzerland), pp. 293-302.

Richter, P. H., Procaccia, I. \& Ross, J. [1981] "Chemical Instabilities" in Advances in Chemical Physics Vol. 43, eds. Prigogine, I, \& Rice, S. (Wiley-Interscience, New York), pp. 217-268.

Sahoo, D. \& Ananthakrishna, G. [1982] "A phenomenological dislocation transformation model for the mobile fraction in simple systems", J. Phys. D 15, 1439-1449.

Stejskalova, V., Hammersky, M., Luckac, P., Vostry, P. \& Spursil, B. [1981] Czech. J. Phys. B31, 195.

Taniuti, T. \& Wei, C. C. [1968] "Reductive perturbative method in nonlinear wave propagation I", J. Phys. Soc. Japan 24, 941-946.

Troger, H. \& Steindl, A. [1991] Nonlinear Stability and Bifurcation Theory (Springer-Verlag, Wien, New York), pp. 52-68.

Valsakumar, M. C. \& Ananthakrishna, G. [1983] "A model based on nonlinear oscillations to explain jumps on creep curves: II. Approximate solutions", J. Phys. D 16, 1055-1068.

Venkadesan, S., Murthy, K. P. N., \& Valsakumar, M. C. [1995] " Chaotic Flow in Al-Mg Alloy" in Solid State Phenomena Vol. 42\&43, eds. Ananthakrishna, G., Kubin, L. P. \& Martin, G. (Scitec, Switzerland), pp. 287-292.

Zagoruyko, L. N., Osetskiy A. I. \& Soldatov, V. P. [1977] "Discontinuous deformation of zinc single crystals under creep conditions", Phys. Met. Metallogr. 43(5), 156-164. 


\section{Appendix A}

In this appendix we give the expressions for the coefficients appearing in the TDGL equation

up to the quintic term, i.e. the expressions for $\mu, \eta$ and $\nu$ in the equation $\frac{d}{d \tau} \Psi=\epsilon \mu \Psi+$ $\eta|\Psi|^{2} \Psi+\nu|\Psi|^{4} \Psi$. They are given by

$$
\begin{gathered}
\mu \equiv \frac{1}{2}\left(c_{0}-\imath \omega\right), \\
\eta \equiv 2 P_{2}\left(k_{1}+k_{2}+k_{2}^{*}+k_{3}^{*}\right)+3 P_{3},
\end{gathered}
$$

and

$$
\nu \equiv 2 P_{2} Q_{2}+3 P_{3} Q_{3}+4 P_{4} Q_{4}+10 P_{5}
$$

where

$$
P_{n} \equiv \frac{\left(a b_{0}\right)^{n-1}}{2 \omega}\left[\omega \xi_{n}-\imath\left(c_{0} \xi_{n}+a b_{0} \zeta_{n}\right)\right]
$$

and

$$
\begin{gathered}
Q_{2} \equiv\left(k_{1}+k_{3}^{*}\right) \ell_{3}+\left(k_{2}+k_{2}^{*}\right) \ell_{3}^{*}+\left(k_{1}^{*}+k_{3}\right)\left(\ell_{2}+\ell_{4}^{*}\right)+m_{5}+m_{6}+m_{6}^{*}+m_{7}^{*} \\
Q_{3} \equiv 2\left(k_{1}+k_{3}^{*}\right)\left(k_{1}^{*}+k_{2}+k_{2}^{*}+k_{3}\right)+\left(k_{2}+k_{2}^{*}\right)^{2}+\ell_{2}+\ell_{3}+2 \ell_{3}^{*}+\ell_{4}^{*} \\
Q_{4} \equiv k_{1}^{*}+k_{3}+3\left(k_{1}+k_{2}+k_{2}^{*}+k_{3}^{*}\right) .
\end{gathered}
$$

The expressions for $\xi_{n}$ 's and $\zeta_{n}$ 's used in Eq.(A5) are given by

$$
\begin{gathered}
\xi_{2} \equiv \frac{b_{0} \chi}{\alpha^{3}}\left[2 \alpha^{2}-a\left(\alpha-b_{0} \chi\right)\right] \\
\xi_{3} \equiv \frac{b_{0} \chi}{\alpha^{5}}\left(\alpha-b_{0} \chi\right)\left(a \Delta-2 \alpha^{2}\right) \\
\xi_{4} \equiv \frac{\alpha^{2}-\Delta^{2}}{16 \alpha^{7}}\left[a\left(\alpha^{2}-5 \Delta^{2}\right)+8 \Delta \alpha^{2}\right] \\
\xi_{5} \equiv-\frac{\alpha^{2}-\Delta^{2}}{16 \alpha^{9}}\left[a \Delta\left(3 \alpha^{2}-7 \Delta^{2}\right)-2 \alpha^{2}\left(\alpha^{2}-\Delta^{2}\right)\right]
\end{gathered}
$$

and

$$
\begin{gathered}
\zeta_{2} \equiv \frac{c_{0}}{4 b_{0} \alpha^{3}}\left(\alpha^{2}-\Delta^{2}\right), \\
\zeta_{3} \equiv-\frac{\Delta}{\alpha^{2}} \zeta_{2} \\
\zeta_{4} \equiv-\frac{\alpha^{2}-5 \Delta^{2}}{4 \alpha^{4}} \zeta_{2}, \\
\zeta_{5} \equiv \frac{\Delta\left(3 \alpha^{2}-7 \Delta^{2}\right)}{4 \alpha^{6}} \zeta_{2},
\end{gathered}
$$

where

$$
\Delta \equiv \alpha-2 b_{0} \chi
$$

Expressions for $k$ 's, $\ell$ 's, $m$ 's and $n$ 's are given in Appendix C. 


\section{Appendix B}

In this appendix the equations describing the secular motion are derived for the case when the order parameter, $\Psi$, is found from the quintic TDGL equation (Eq. 44).

From Eq. (39),

$$
Y(\tau)=a b_{0}\left(\Psi e^{\imath \omega \tau}+\text { c.c. }\right)
$$

and

$$
Z(\tau)=\left(-c_{0}+\imath \omega\right) \Psi e^{\imath \omega \tau}+\text { c.c. }
$$

where the complex amplitude is $\Psi=\epsilon^{\frac{1}{2}} \psi_{1}+\epsilon \psi_{2}+\ldots+\epsilon^{\frac{5}{2}} \psi_{5}$ with $\psi_{2}, \ldots, \psi_{5}$ given in terms of functions of $\psi_{1}$ and phase factors (see Appendix C). On the other hand, to $\mathcal{O}\left(\epsilon^{\frac{1}{2}}\right)$,

$$
\Psi=\epsilon^{\frac{1}{2}} \psi_{1},
$$

which, upon inverting, gives $\psi_{1}$ in terms of $\Psi$ :

$$
\psi_{1}=\epsilon^{-\frac{1}{2}}|\Psi| e^{\imath \Omega \tau} .
$$

Substituting the $\psi_{2}, \ldots, \psi_{5}$-expressions from Appendix C in Eqs. (B1) and (B2) and, then, using Eq. (B4) leads to obtaining the secular equations given by:

$$
Y=2 a b_{0} P_{+}
$$

and

$$
Z=-2 c_{0} P_{+}-2 \omega P_{-}
$$

where

$$
\begin{aligned}
P_{ \pm} \equiv & |\Psi| F_{ \pm}\left(\Omega_{c} \tau\right)+K_{13}^{ \pm}|\Psi|^{2} F_{ \pm}\left(2 \Omega_{c} \tau+\theta_{k_{13}}^{ \pm}\right)+|\Psi|^{2} G_{ \pm}\left(k_{2}\right) \\
& +\epsilon L_{1}|\Psi| F_{ \pm}\left(\Omega_{c} \tau-\theta_{\ell_{1}}\right)+L_{24}^{ \pm}|\Psi|^{3} F_{ \pm}\left(3 \Omega_{c} \tau+\theta_{\ell_{24}}^{ \pm}\right)+L_{3}|\Psi|^{3} F_{ \pm}\left(\Omega_{c} \tau-\theta_{\ell_{3}}\right) \\
& +\epsilon M_{13}^{ \pm}|\Psi|^{2} F_{ \pm}\left(2 \Omega_{c} \tau+\theta_{m_{13}}^{ \pm}\right)+\epsilon|\Psi|^{2} G_{ \pm}\left(m_{2}\right)+M_{48}^{ \pm}|\psi|^{4} F_{ \pm}\left(4 \Omega_{c} \tau+\theta_{m_{48}}^{ \pm}\right) \\
& +M_{57}^{ \pm}|\Psi|^{4} F_{ \pm}\left(2 \Omega_{c} \tau+\theta_{m_{57}}^{ \pm}\right)+|\psi|^{4} G_{ \pm}\left(m_{6}\right)+\epsilon^{2} N_{1}|\Psi| F_{ \pm}\left(\Omega_{c} \tau-\theta_{n_{1}}\right) \\
& +\epsilon N_{24}^{ \pm}|\Psi|^{3} F_{ \pm}\left(3 \Omega_{c} \tau+\theta_{n_{24}}^{ \pm}\right)+\epsilon N_{3}|\Psi|^{3} F_{ \pm}\left(\Omega_{c} \tau-\theta_{n_{3}}\right)+N_{59}^{ \pm}|\Psi|^{5} F_{ \pm}\left(5 \Omega_{c} \tau+\theta_{n_{59}}^{ \pm}\right) \\
& +N_{68}^{ \pm}|\Psi|^{5} F_{ \pm}\left(3 \Omega_{c} \tau+\theta_{n_{68}}^{ \pm}\right)+N_{7}^{ \pm}|\Psi|^{5} F_{ \pm}\left(\Omega_{c} \tau-\theta_{n_{7}}^{ \pm}\right)
\end{aligned}
$$

with

$$
\begin{aligned}
F_{+}(\phi) & \equiv \cos (\phi), \\
F_{-}(\phi) & \equiv \sin (\phi), \\
G_{+}(u) & \equiv \operatorname{Real}(u) \\
G_{-}(u) & \equiv \operatorname{Im}(u), \\
\Omega_{c} & \equiv \omega+\Omega \\
V_{i j}^{ \pm} & \equiv\left|v_{i} \pm v_{j}^{\star}\right| \\
V_{i} & \equiv\left|v_{i}\right| \\
\Theta_{v_{i j}}^{ \pm} & \equiv \sin ^{-1}\left(\frac{\operatorname{Real}\left(v_{i} \pm v_{j}^{\star}\right)}{\left|v_{i} \pm v_{j}^{\star}\right|}\right)
\end{aligned}
$$

where $V$ stands for either $K, L, M$ or $N$, while $v$ stands for either $k, \ell, m$, or $n$ correspondingly. Expressions for $K, L, M$ and for $N$ can be obtained from $k$ 's, $\ell$ 's, $m$ 's and $n$ 's which are given in Appendix C. 


\section{Appendix C}

Expressions for $\psi_{2}, \psi_{3}, \psi_{4}, \psi_{5}$ (with a factor $e^{\imath \omega \tau}$ ) are

$$
\begin{aligned}
\psi_{2} e^{i \omega \tau}= & k_{1} \psi_{1}^{2} e^{2 i \omega \tau}+k_{2}\left|\psi_{1}\right|^{2}+k_{3} \psi_{1}^{\star^{2}} e^{-2 i \omega \tau} \\
\psi_{3} e^{i \omega \tau}= & \ell_{1} \psi_{1}^{\star} e^{-i \omega \tau}+\ell_{2} \psi_{1}^{3} e^{3 i \omega \tau}+\ell_{3}\left|\psi_{1}\right|^{2} \psi_{1}^{\star} e^{-i \omega \tau}+\ell_{4} \psi_{1}^{\star^{3}} e^{-3 i \omega \tau} \\
\psi_{4} e^{i \omega \tau}= & m_{1} \psi_{1}^{2} e^{2 i \omega \tau}+m_{2}\left|\psi_{1}^{2}\right|+m_{3} \psi_{1}^{\star^{2}} e^{-2 i \omega \tau}+m_{4} \psi_{1}^{4} e^{4 i \omega \tau}+m_{5}\left|\psi_{1}\right|^{2} \psi_{1}^{2} e^{2 i \omega \tau} \\
& +m_{6}\left|\psi_{1}\right|^{4}+m_{7}\left|\psi_{1}\right|^{2} \psi_{1}^{\star^{2}} e^{-2 i \omega \tau}+m_{8} \psi_{1}^{\star^{4}} e^{-4 i \omega \tau} \\
\psi_{5} e^{2 i \omega \tau}= & n_{1} \psi_{1}^{\star} e^{-i \omega \tau}+n_{2} \psi_{1}^{3} e^{3 i \omega \tau}+n_{3}\left|\psi_{1}\right|^{2} \psi_{1}^{\star} e^{-i \omega \tau}+n_{4} \psi_{1}^{\star^{3}} e^{-3 i \omega \tau}+n_{5} \psi_{1}^{5} e^{5 i \omega \tau} \\
& +n_{6}\left|\psi_{1}\right|^{2} \psi_{1}^{3} e^{3 i \omega \tau}+n_{7}\left|\psi_{1}\right|^{4} \psi_{1}^{\star} e^{-i \omega \tau}+n_{8}\left|\psi_{1}\right|^{2} \psi_{1}^{\star^{3}} e^{-3 i \omega \tau}+n_{9} \psi_{1}^{\star^{5}} e^{-5 i \omega \tau}
\end{aligned}
$$

where

$$
\begin{aligned}
& k_{1} \equiv-\frac{i}{\omega} P_{2} \\
& k_{2} \equiv \frac{2 i}{\omega} P_{2} \\
& k_{3} \equiv \frac{i}{3 \omega} P_{2} \text {, } \\
& \ell_{1} \equiv-\frac{i}{2 \omega} \mu^{\star} \\
& \ell_{2} \equiv-\frac{i}{2 \omega}\left[2 P_{2}\left(k_{1}+k_{3}^{\star}\right)+P_{3}\right] \text {, } \\
& \ell_{3} \equiv \frac{i}{2 \omega}\left[2 P_{2}\left(k_{1}^{\star}+k_{2}^{\star}+k_{2}+k_{3}\right)+3 P_{3}\right] \text {, } \\
& \ell_{4} \equiv \frac{i}{4 \omega}\left[2 P_{2}\left(k_{1}^{\star}+k_{3}\right)+P_{3}\right] \\
& m_{1} \equiv-\frac{i}{\omega}\left[-\mu k_{1}-\mu^{\star} k_{3}^{\star}+2 P_{2} \ell_{1}^{\star}\right], \\
& m_{2} \equiv \frac{i}{\omega}\left[-\mu^{\star}\left(k_{2}+k_{2}^{\star}\right)+2 P_{2}\left(\ell_{1}+\ell_{1}^{\star}\right)\right] \text {, } \\
& m_{3} \equiv \frac{i}{3 \omega}\left[\mu k_{3}-\mu^{\star}\left(k_{1}^{\star}+2 k_{3}\right)+2 P_{2} \ell_{1}\right] \text {, } \\
& m_{4} \equiv-\frac{i}{3 \omega}\left\{P_{2}\left[\left(k_{1}+k_{3}^{\star}\right)^{2}+2\left(\ell_{2}+\ell_{4}^{\star}\right)\right]+3 P_{3}\left(k_{1}+k_{3}^{\star}\right)+P_{4}\right\}, \\
& m_{5} \equiv-\frac{i}{\omega}\left\{2 P_{2}\left[\left(k_{1}+k_{3}^{\star}\right)\left(k_{2}+k_{2}^{\star}\right)+\ell_{2}+\ell_{3}^{\star}+\ell_{4}^{\star}\right]+3 P_{3}\left[2\left(k_{1}+k_{3}^{\star}\right)+k_{2}+k_{2}^{\star}\right]\right. \\
& \left.+4 P_{4}-2 k_{1} \eta\right\} \\
& m_{6} \equiv \frac{i}{\omega}\left\{P_{2}\left[2\left(k_{1}+k_{3}^{\star}\right)\left(k_{1}^{\star}+k_{3}\right)+\left(k_{2}+k_{2}^{\star}\right)^{2}+2\left(\ell_{3}+\ell_{3}^{\star}\right)\right]\right. \\
& \left.+3 P_{3}\left[k_{1}+k_{1}^{\star}+2\left(k_{2}+k_{2}^{\star}\right)+k_{3}+k_{3}^{\star}\right]+6 P_{4}-2 k_{2} \eta_{1}\right\} \\
& m_{7} \equiv \frac{i}{3 \omega}\left\{2 P_{2}\left[\left(k_{1}^{\star}+k_{3}\right)\left(k_{2}^{\star}+k_{2}\right)+\ell_{2}^{\star}+\ell_{3}+\ell_{4}\right]+3 P_{3}\left[2\left(k_{1}^{\star}+k_{3}\right)+k_{2}+k_{2}^{\star}\right]\right. \\
& \left.+4 P_{4}-2 k_{3} \eta^{\star}\right\} \\
& m_{8} \equiv \frac{i}{5 \omega}\left\{P_{2}\left[\left(k_{1}^{\star}+k_{3}\right)^{2}+2\left(\ell_{2}^{\star}+\ell_{4}\right)\right]+3 P_{3}\left(k_{1}^{\star}+k_{3}\right)+P_{4}\right\} \text {, } \\
& n_{1} \equiv \frac{i}{2 \omega}\left(\mu-\mu^{\star}\right) \ell_{1} \text {, }
\end{aligned}
$$




$$
\begin{aligned}
& n_{2} \equiv-\frac{i}{2 \omega}\left\{2 P_{2}\left[\ell_{1}^{\star}\left(k_{1}+k_{3}^{\star}\right)+m_{1}+m_{3}^{\star}\right]+3 P_{3} \ell_{1}^{\star}-2 \mu \ell_{2}-\mu^{\star} \ell_{4}^{\star}\right\} \\
& n_{3} \equiv \frac{i}{2 \omega}\left\{2 P_{2}\left[\ell_{1}\left(k_{2}+k_{2}^{\star}\right)+\ell_{1}^{\star}\left(k_{1}^{\star}+k_{3}\right)+m_{1}^{\star}+m_{2}^{\star}+m_{2}+m_{3}\right]\right. \\
&\left.+3 P_{3}\left(\ell_{1}^{\star}+2 \ell_{1}\right)-\eta^{\star} \ell_{1}-2 \mu^{\star} \ell_{3}\right\} \\
& n_{4} \equiv \frac{i}{4 \omega}\left\{2 P_{2}\left[\ell_{1}\left(k_{1}^{\star}+k_{3}\right)+m_{1}^{\star}+m_{3}\right]+3 P_{3} \ell_{1}-\mu^{\star} \ell_{2}^{\star}+\left(\mu-3 \mu^{\star}\right) \ell_{4}\right\} \\
& n_{5} \equiv-\frac{i}{4 \omega}\left\{2 P_{2}\left[m_{4}+m_{8}^{\star}+\left(k_{1}+k_{3}^{\star}\right)\left(\ell_{2}+\ell_{4}^{\star}\right)\right]+3 P_{3}\left[\left(k_{1}+k_{3}^{\star}\right)^{2}+\ell_{2}+\ell_{4}^{\star}\right]\right. \\
&\left.+4 P_{4}\left(k_{1}+k_{3}^{\star}\right)+P_{5}\right\} \\
& n_{6} \equiv-\frac{i}{2 \omega}\left\{2 P_{2}\left[m_{4}+m_{5}+m_{7}^{\star}+m_{8}^{\star}+\ell_{3}^{\star}\left(k_{1}+k_{3}^{\star}\right)+\left(\ell_{2}+\ell_{4}^{\star}\right)\left(k_{2}+k_{2}^{\star}\right)\right]\right. \\
&+3 P_{3}\left[\ell_{3}^{\star}+2\left(\ell_{2}+\ell_{4}^{\star}\right)+\left(k_{1}+k_{3}^{\star}\right)^{2}+2\left(k_{1}+k_{3}^{\star}\right)\left(k_{2}+k_{2}^{\star}\right)\right] \\
&\left.+4 P_{4}\left[k_{2}+k_{2}^{\star}+3\left(k_{1}+k_{3}^{\star}\right)\right]+5 P_{5}-3 \eta \ell_{2}\right\} \\
& n_{7} \equiv \frac{i}{2 \omega}\left\{2 P_{2}\left[m_{5}^{\star}+m_{6}^{\star}+m_{6}+m_{7}+\left(\ell_{2}^{\star}+\ell_{4}\right)\left(k_{1}+k_{3}^{\star}\right)+\ell_{3}\left(k_{2}+k_{2}^{\star}\right)+\ell_{3}^{\star}\left(k_{1}^{\star}+k_{3}\right)\right]\right. \\
&+3 P_{3}\left[\left(k_{2}+k_{2}^{\star}\right)^{2}+2\left(k_{1}^{\star}+k_{3}\right)\left(k_{1}+k_{2}+k_{2}^{\star}+k_{3}^{\star}\right)+\ell_{2}^{\star}+\ell_{3}^{\star}+2 \ell_{3}+\ell_{4}\right] \\
&\left.+4 P_{4}\left[k_{1}+k_{3}^{\star}+3\left(k_{1}^{\star}+k_{2}^{\star}+k_{2}+k_{3}\right)\right]+10 P_{5}-\left(\eta+2 \eta^{\star}\right) \ell_{3}\right\} \\
& n_{9} \equiv \frac{i}{6 \omega}\left\{2 P _ { 2 } \left[m_{4}^{\star}+m_{8}+\left(\ell_{2}^{\star}+\ell_{4}\right)\left(k_{1}^{\star}+k_{3}\right]+3 P_{3}\left[\left(k_{1}^{\star}+k_{3}\right)^{2}+\ell_{4}^{\star}+\ell_{4}\right]\right.\right. \\
&\left.+4 P_{4}\left(k_{1}^{\star}+k_{3}\right)+P_{5}\right\} \\
& n_{8}\left[2 P_{2}\left[m_{4}^{\star}+m_{5}^{\star}+m_{7}+m_{8}+\ell_{3}\left(k_{1}^{\star}+k_{3}\right)+\left(\ell_{2}^{\star}+\ell_{4}\right)\left(k_{2}+k_{2}^{\star}\right)\right]\right. \\
&+3 P_{3}\left[\left(k_{1}^{\star}+k_{3}\right)^{2}+2\left(k_{2}+k_{2}^{\star}\right)\left(k_{1}^{\star}+k_{3}\right)+2\left(\ell_{2}^{\star}+\ell_{4}\right)+\ell_{3}\right] \\
&\left.+4 P_{4}\left[k_{2}+k_{2}^{\star}+3\left(k_{1}^{\star}+k_{3}\right)\right]+5 P_{5}-3 \eta^{\star} \ell_{4}\right\}
\end{aligned}
$$

Note that expressions for $P_{2}, \ldots, P_{5}$ are given in Eq. (A5) in Appendix A. 


\section{Figure Captions}

Fig. 1 Plot of $\frac{1}{c_{0}}$ versus $a$ with $b_{0}=10^{-4}$. The hatched portion shows the instability region which extends between $a=\frac{1}{3}$ and $a=\frac{1}{\sqrt{2}}$ approximately.

Fig. 2 Plots of $\eta_{1}$ and $\nu_{1}$ versus $a\left(b_{0}=10^{-4}\right)$. Note that $\eta_{1}$ is negative (but finite) in the interval $0.4713 \leq a \leq 0.5210$. Similarly, $\nu_{1}$ is negative (but finite) in the interval $0.4538 \leq a \leq 0.5097$.

Fig. 3 Plots of $|\Psi|^{2}$ and $P$ versus $a$ in the region of supercritical bifurcation.

Fig. 4 Plots of $|\Psi|^{2}$ and $P$ versus $a$ in a subregion of subcritical bifurcation, i. e. the region where quintic TDGL equation holds.

Fig. 5 Plots of the (numerical) limit cycle solutions obtained from the secular equations (dotted line), the reduced model (dashed line) and the $Y^{3}$-truncated model (crossed line) for $a=0.5$ and $\epsilon=10^{-4}$.

Fig. 6 Plots of the (numerical) limit cycle solutions obtained from the secular equations (dotted line), the reduced model (dashed line) and the $Y^{5}$-truncated model (crossed line) for $a=0.468$ and $\epsilon=10^{-4}$.

Fig. 7 Plots of the (numerical) limit cycle solutions obtained from the reduced model (dashed line) and the $Y^{7}$-truncated model (crossed line) for $a=0.44$ and $\epsilon=10^{-4}$.

Fig. 8 Plot of the (numerical) limit cycle solution obtained from the reduced model for $a=0.63$ and $\epsilon=10^{-4}$. 


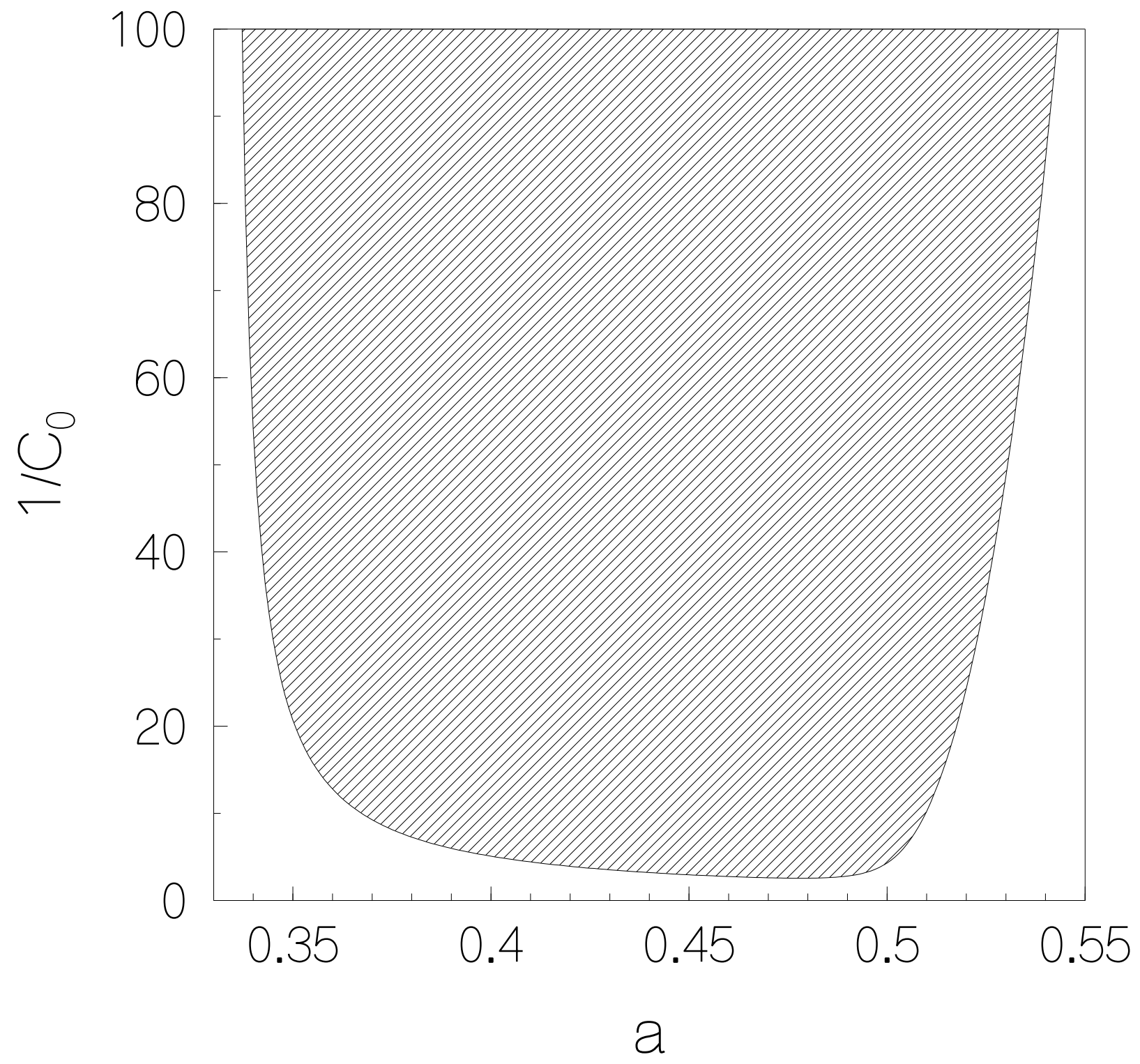




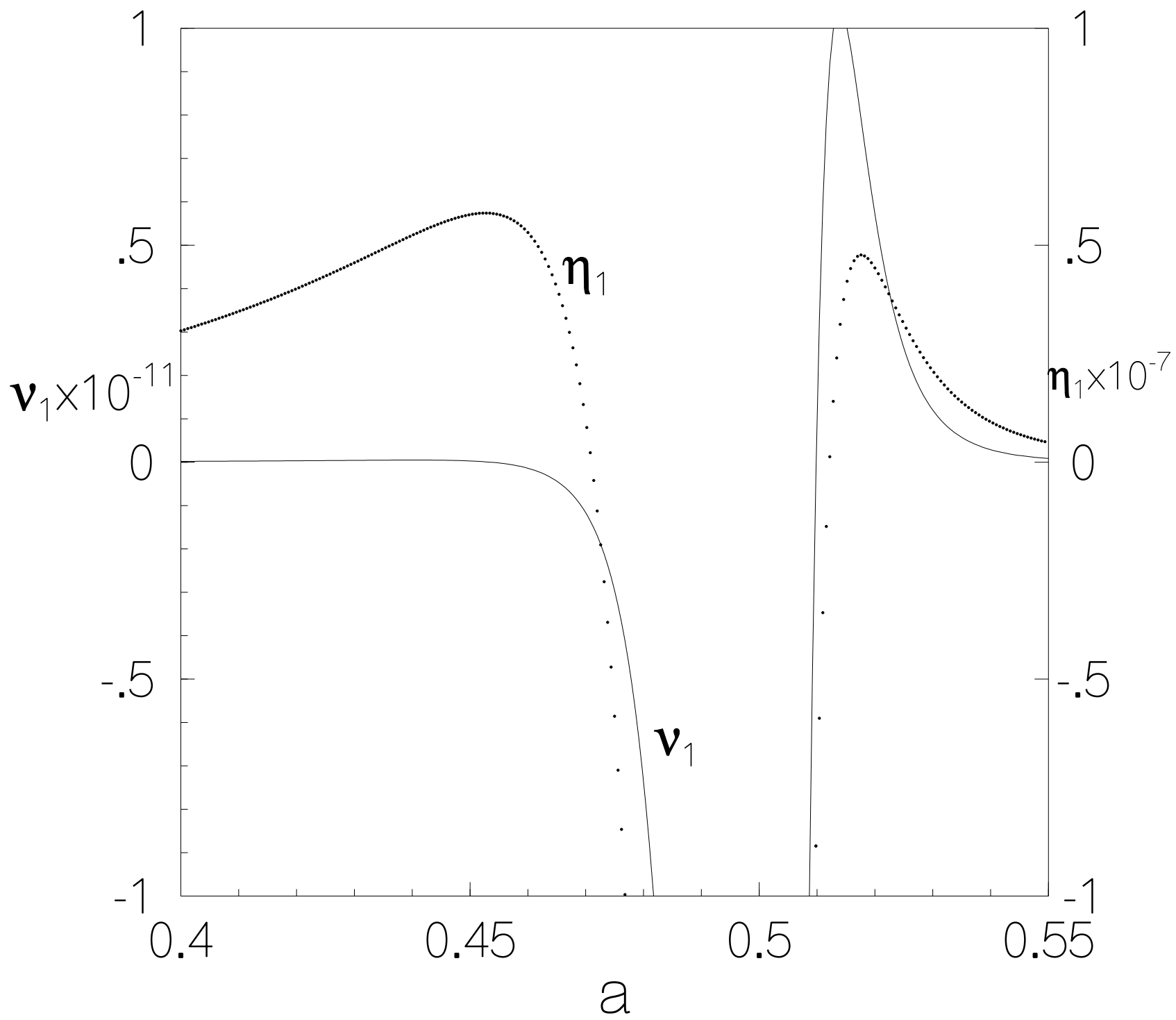




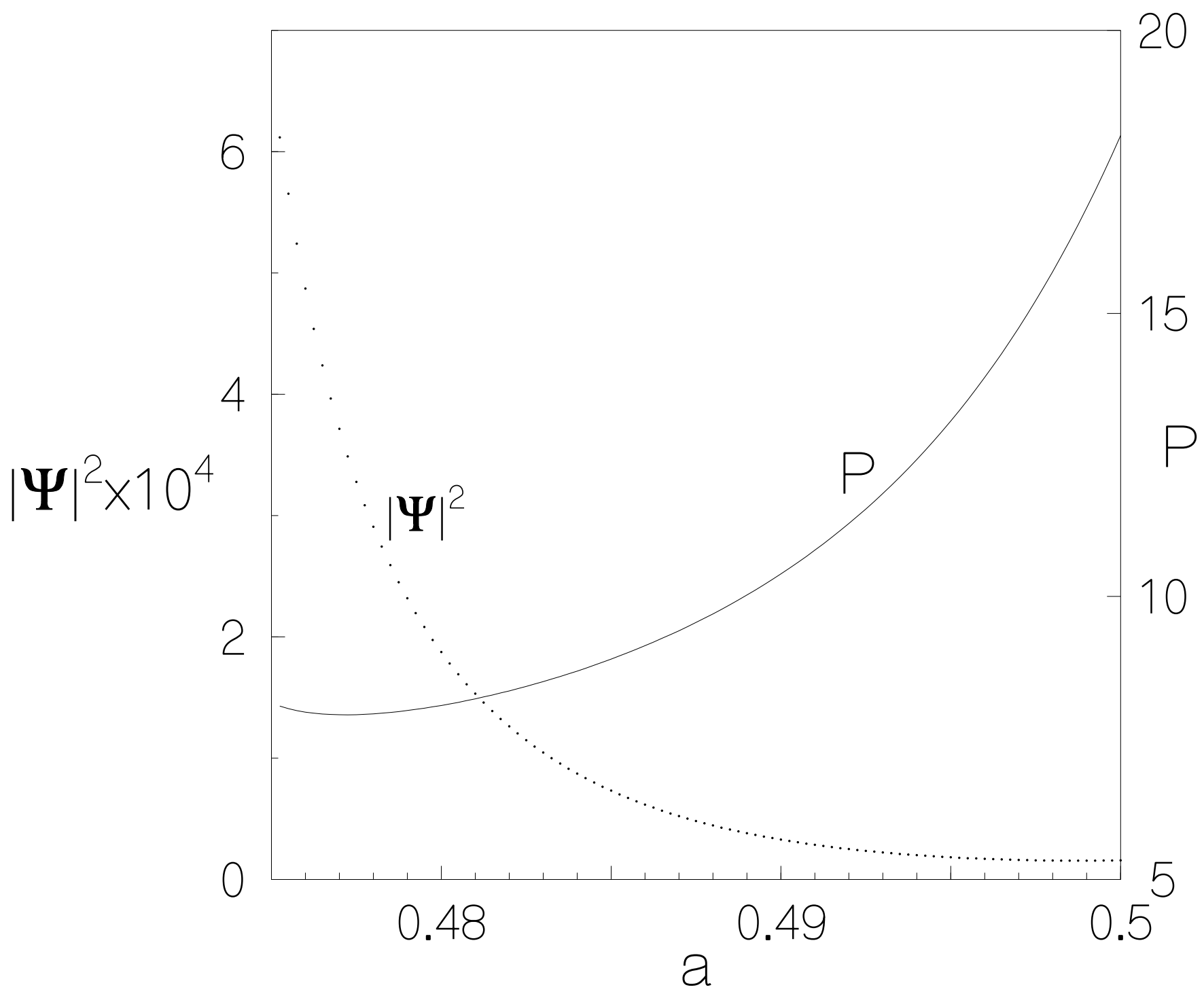




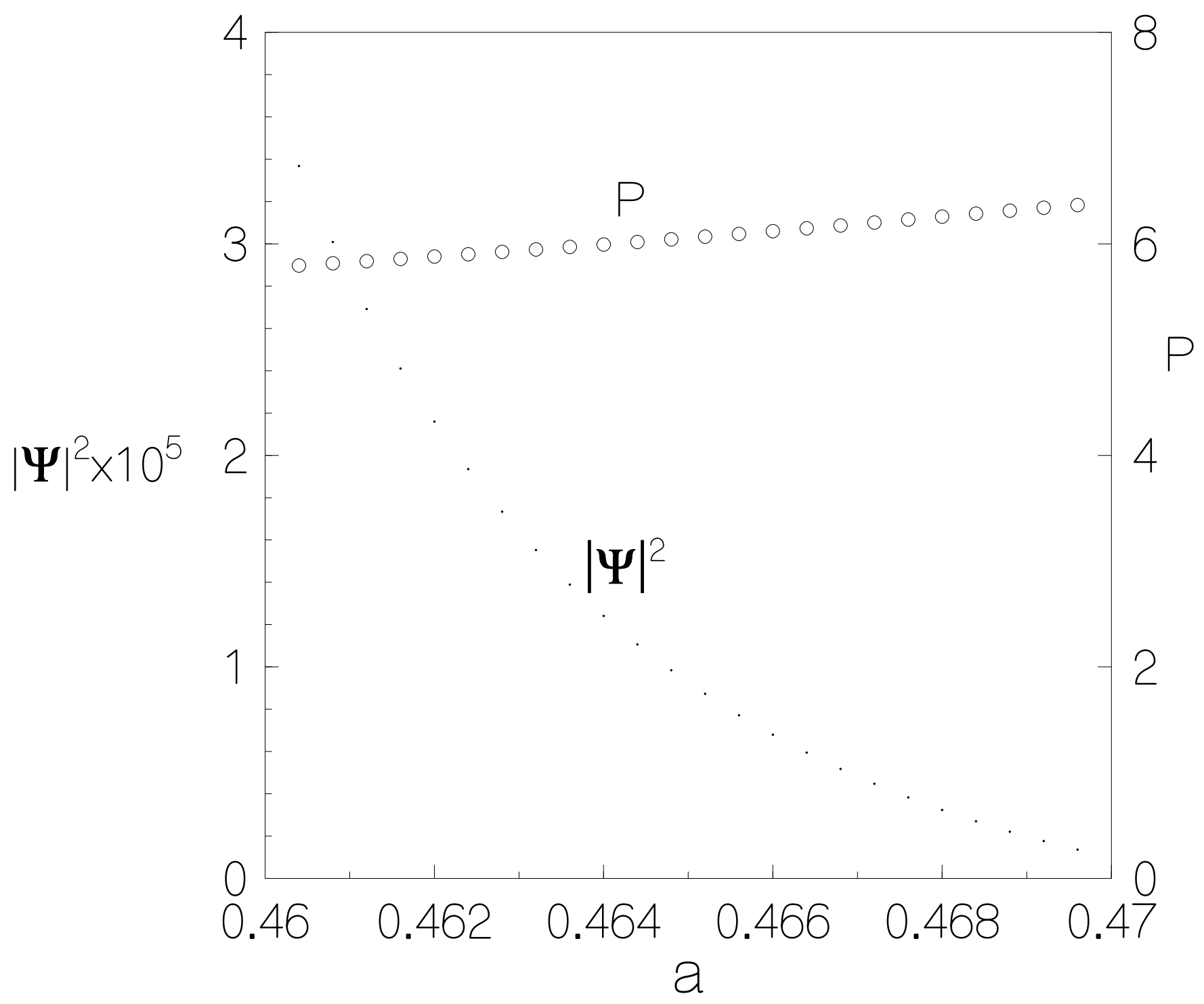




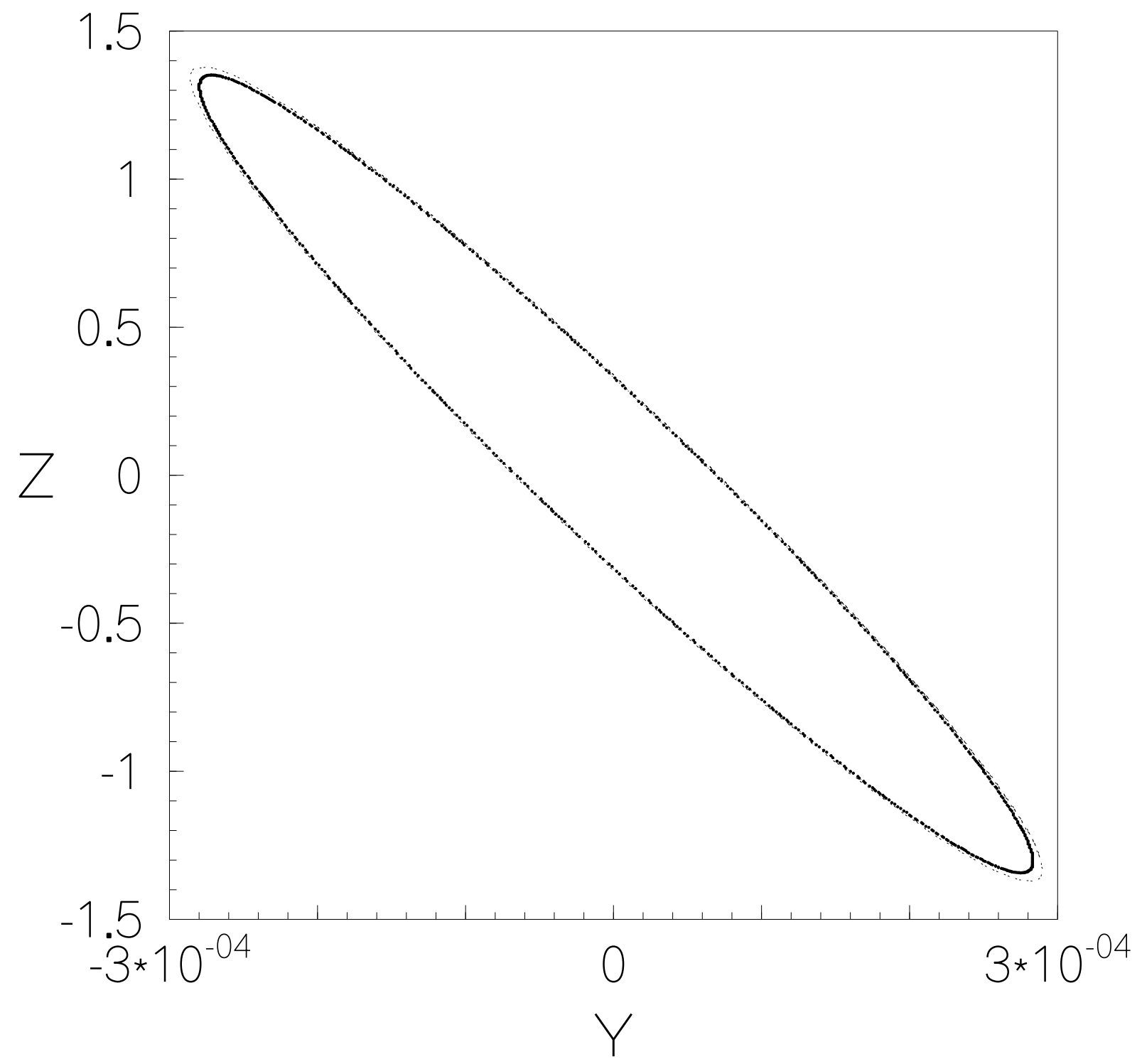




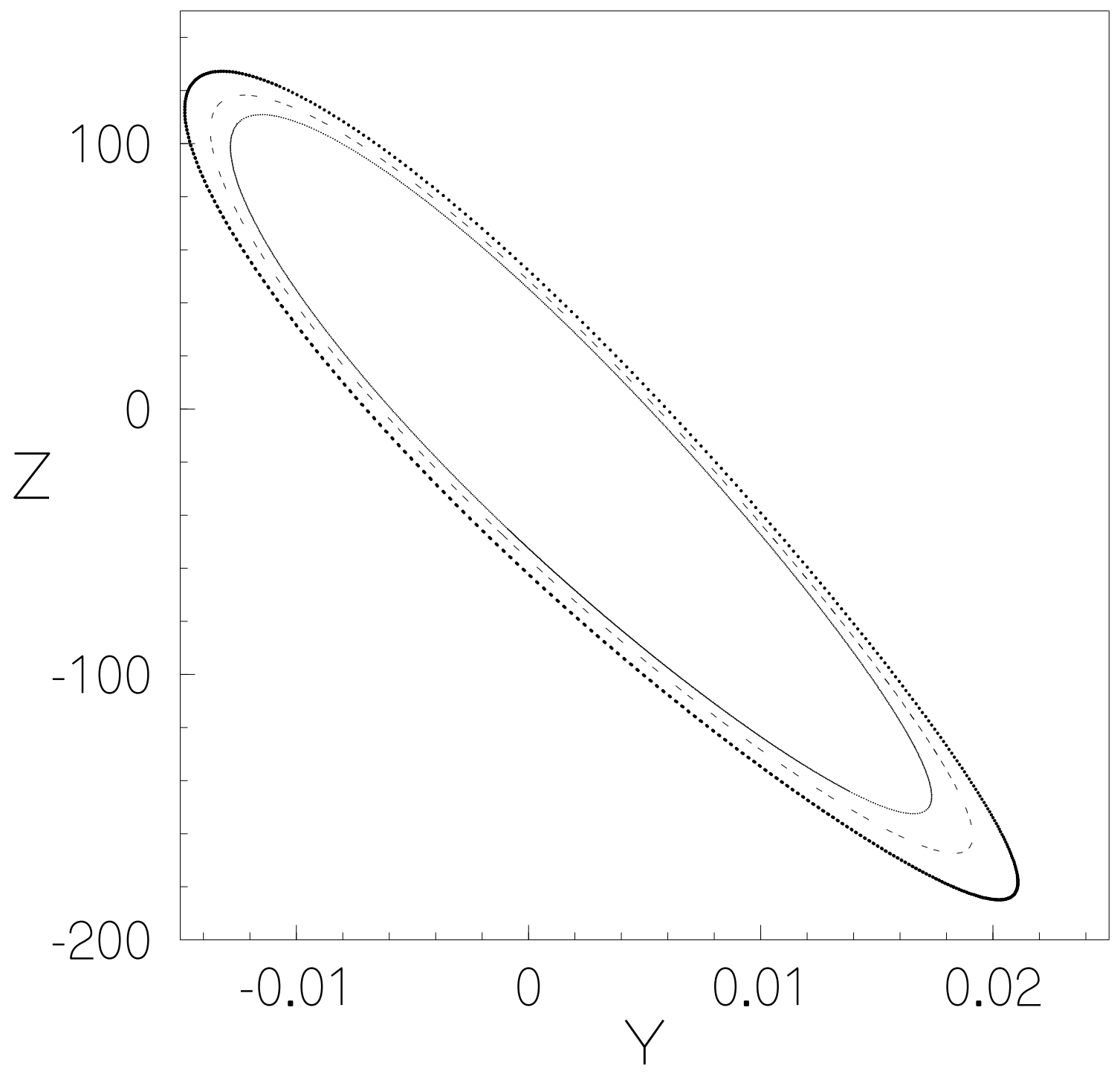




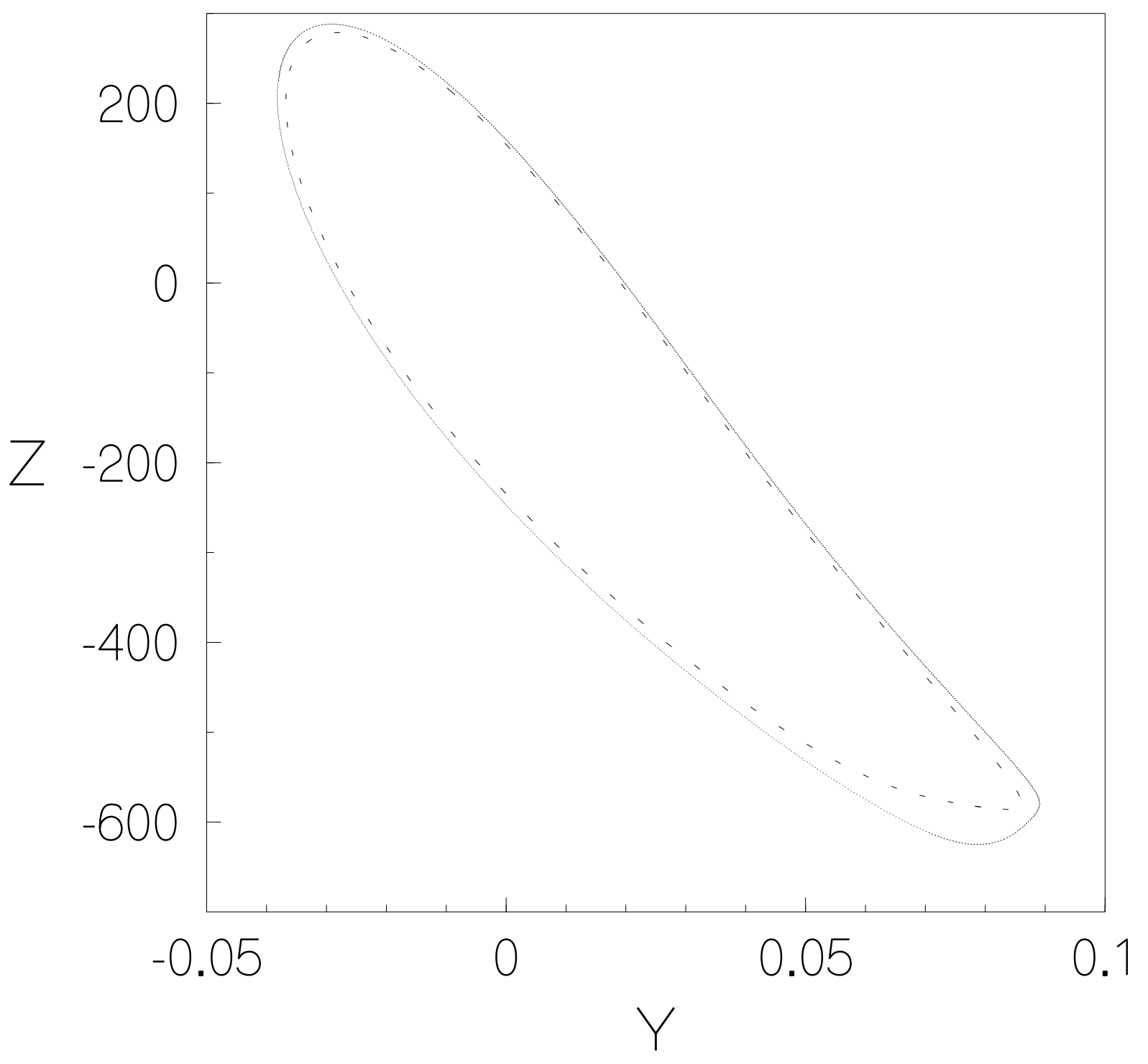




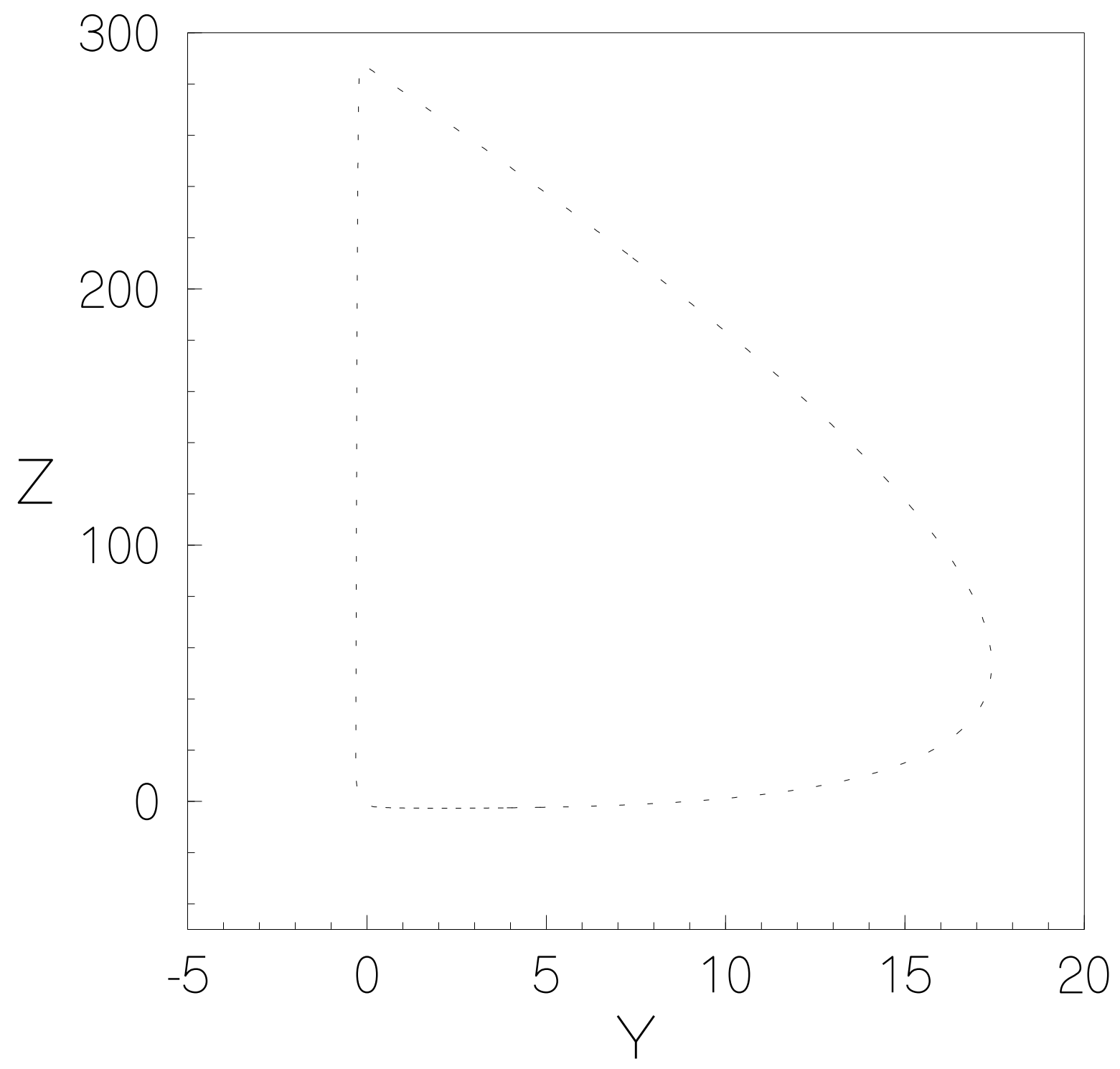

